Document downloaded from:

http://hdl.handle.net/10251/153228

This paper must be cited as:

Miralles Ricós, R.; Lara Martínez, G.; Gosálbez Castillo, J.; Bosch Roig, I.; León Fernández, A. (2019). Improved visualization of large temporal series for the evaluation of good environmental status. Applied Acoustics. 148:55-61.

https://doi.org/10.1016/j.apacoust.2018.12.009

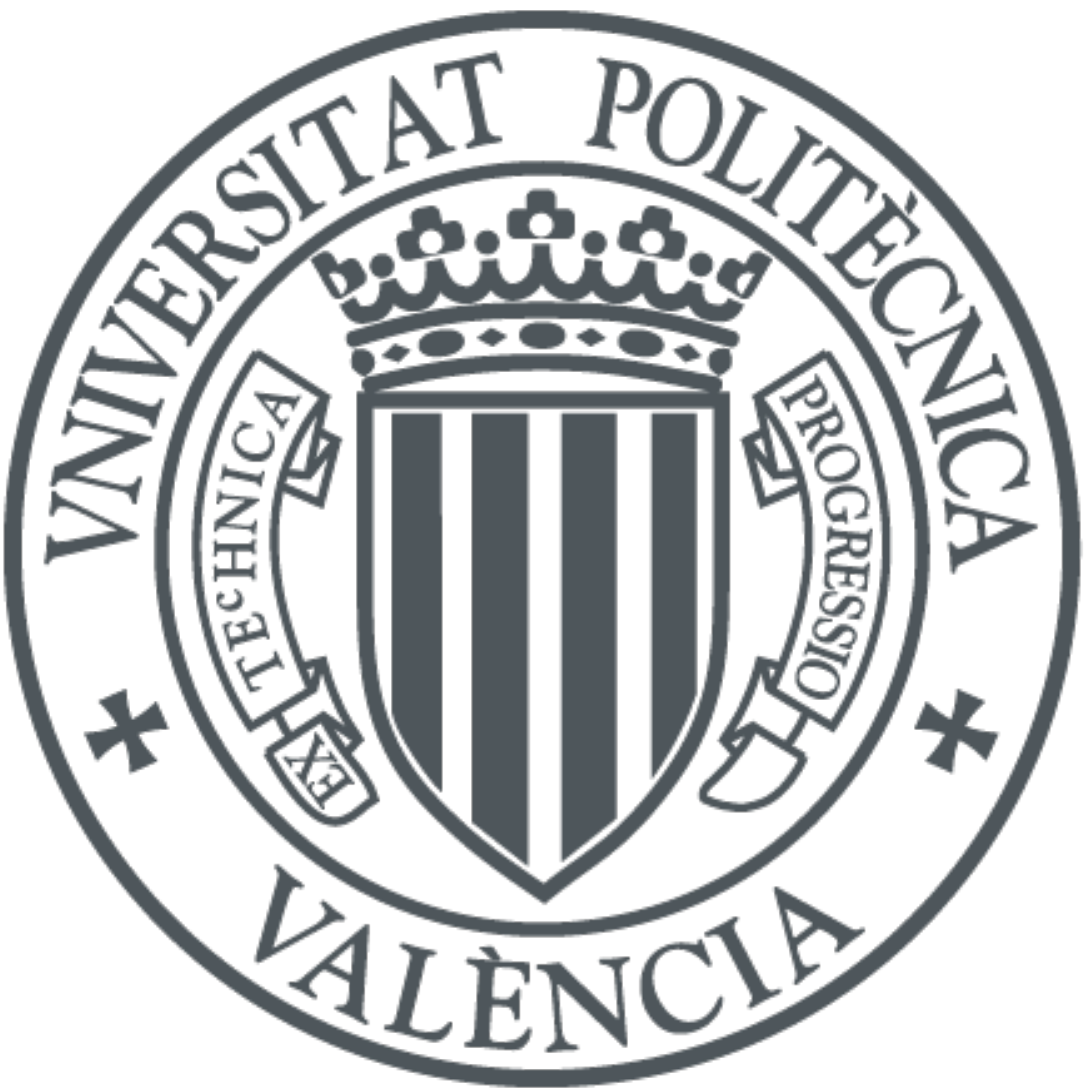

The final publication is available at

https://doi.org/10.1016/j.apacoust.2018.12.009

Copyright Elsevier

Additional Information 


\title{
Improved Visualization of Large Temporal Series for the Evaluation of Good Environmental Status
}

\author{
Ramón Miralles $^{\mathrm{a}, \mathrm{b}, *}$, Guillermo Lara ${ }^{\mathrm{a}}$, Jorge Gosalbez ${ }^{\mathrm{a}, \mathrm{b}}$, Ignacio Bosch ${ }^{\mathrm{a}, \mathrm{b}}$, Antonio León ${ }^{\mathrm{b}}$, \\ ${ }^{a}$ Institute of Telecommunications and Multimedia Applications (iTEAM), Universitat Politècnica de València, Spain \\ ${ }^{b}$ Departamento de Comunicaciones, Universitat Politècnica de València, Spain
}

\begin{abstract}
Passive acoustic monitoring of underwater sounds is an emerging discipline that can be used to guarantee that anthropogenic noise meets acceptable limits, to detect the presence of cetacean species, and to ensure sustainable exploitation of our oceans and seas. In this scenario, graphical representation techniques play a key role in helping to reveal seasonal structures of human made noises. Nevertheless, for very long temporal series, it might be challenging to find a graphic visualization technique that allows representing a time range that is long enough to capture these seasonal events, while at the same time preserving short isolated events. We propose a framework for the creation of such visualization techniques and analyze the different stages involved: data reduction, color encoding, and signal processing on graphs. All of this is applied to data from deployments in two marine protected areas in order to provide an acoustic panorama and identify seasonal events.
\end{abstract}

Keywords: Big Data Visualization, Acoustic Images, Ambient Noise Characterization, Submarine Environment, Surveillance, Data Reduction, Hough Transform

\section{Introduction}

Anthropogenic sources of noise in the marine environment have increased in the last few decades. This is mainly due to the expansion in shipping, oil and gas exploration, infrastructure development, etc. These noises of different intensity and frequency can result in chronic and acute effects on marine organisms (e.g., $[1,2])$. With the aim of more effectively protecting the marine environment across Europe, the Marine Strategy Framework Directive (MSFD) was adopted in June 2008. The MSFD aims to achieve Good Environmental Status (GES) of the EU's marine waters by 2020 and to protect the resource base upon which marine-related economic and social activities depend. Among many other descriptors, Descriptor 11 covers the assessment of noise (as well as other forms of energy) and gives indications on how to measure the impulsive and ambient noise. In the case of the ambient noise, Sound Pressure Level (SPL) indicators are defined and can be obtained as described in Eq. (1),

${ }^{*}$ Corresponding author at: Universitat Politècnica de València iTEAM, Camino de Vera, S/N, 46022, Valencia, Spain

Email address: rmiralle@dcom.upv.es (Ramón Miralles)

$$
S P L_{1 / 3}=10 \cdot \log _{10} \frac{\frac{1}{T} \int_{0}^{T} p_{1 / 3}^{2}(t) d t}{p_{0}^{2}}
$$

where $p_{1 / 3}(t)$ is the sound pressure in $[\mathrm{Pa}]$ in the desired $1 / 3$ octave band and $T$ is the integration time in [sec]. The indicator, $S P L_{1 / 3}$, is given in logarithmic units relative to the square value of some reference sound pressure $p_{0}$ in [Pa]. Typically, $63 \mathrm{~Hz}, 125 \mathrm{~Hz}$, and $2000 \mathrm{~Hz}$ are the common 1/3 octave bands employed and $p_{0}$ is $1 \mu P a$ in underwater acoustics. The first two bands $(63 \mathrm{~Hz}$ and $125 \mathrm{~Hz})$ are mandatory in the MSFD, but the third band, though not obligatory, is frequently used since ambient noise also peaks at higher frequency levels ( $>1 \mathrm{kHz})[3]$.

A great amount of work has been done to study how SPL indicators can be used in the definition of a GES. Recently, many authors have adressed the problem of setting ambient noise thresholds for environmnetal status asessment (e.g., $[4,5])$. This is being done from different perspectives: population level and displacement of species, bioacoustics, etc. Although many advances have been made, this is still an open problem that requires new and enhanced analysis tools such as big data visualization techniques. Additionally, some of these 
thresholds need to be updated regularly as techniques evolve, which makes the problem even more complex. For this purpose, it is important to devise specific and enhanced graphical representation techniques to allow the representation of ambient noise $1 / 3$ octave indicators. Improved visualization techniques need to take into account the very special characteristics of acoustic impact studies. There are two main features a suitable visualization technique should have: the possibility of representing very long temporal series and the visual identification of seasonal structures (hourly, daily, etc.). It is important to highlight that some of the recorders remain underwater for long periods of time and that the information provided can cover several months ("Big Data" problem). Traditional data visualization tools are often inadequate to handle big data due to the fact that visualizing every data point can lead to over-plotting and can produce a graph which is far too cluttered to perceive any trends, anomalies or structure [6]. With regard to visual identification of seasonal structures, many different strategies for the arrangement of the temporal variable at different scales can be chosen, each of which is interesting for highlighting different types of seasonal and cyclic patterns.

In addition to the $1 / 3$ octave band indicators, many authors are extracting information about the presence of some cetacean species with the help of machine learning techniques (e.g., [7, 8, 9, 10]). The new representation framework should allow the visualization of all of this information ( $1 / 3$ octave band indicators and species detection) in such a way that perceptual and interactive scalability of the visualized data is limited by the chosen resolution and not by the number of records [11].

The remainder of this work is structured as follows. In Section 2, we present a visualization technique that is very appropriate for the representation of underwater noise indicators of very long temporal series. Signal processing techniques and data reduction methods can be used in these graphs to ensure that no interesting structures or outliers are missed, to help spot repetitive patterns, and to show the presence of different cetacean species as graphic layers. In Section 3, we introduce a different type of graph that is specifically designed to show the cyclical nature of certain events and how this cyclic nature is reflected in the $1 / 3$ octave noise indicators. Finally, Section 4 presents our conclusions and future work.

\section{Long time series representation of ambient noise indicators}

Line graphs are the simplest way to represent time series data. They are intuitive, easy to create, and give a quick sense of the trend over time. However, there are some drawbacks with this kind of representation when dealing with very long temporal series. The first drawback is that visualizing data points in a linear plot may not be the best idea when seasonality in data at different observation scales must be identified. Two dimensional representations such as (Hour, Day) or (Minutes, Day) are more convenient for this purpose. Secondly, trying to visualize every data point can overwhelm our perceptual capacities and lead to over-plotting due to limitations in the resolution of conventional displays. For instance, using an inadequate representation technique could lead to undetected seasonal patterns such as those produced by recreational activities as well as to failure in detecting abnormal anthropogenic noises of high intensity.

\subsection{Heat maps}

Geospatial visualizations often use heat maps since they quickly allow the identification of "hot spots" or regions of high concentrations of a given variable. Although less frequently used, heat maps can also be used to represent temporal series if the data are conveniently structured in 2D time frames (Hour, Day). This representation technique is convenient when large temporal series need to be represented, as frequently happens with underwater noise indicators. For instance, we can use 24 hours in a day (or 1440 minutes) spread across 30-31 days of the month or 7 days of the week. The process of obtaining the 2D representation from 1D underwater audio recordings is schematically illustrated in Figure 1. First, we need to process the recordings and extract the parameter to be represented (e.g., $S P L_{1 / 3}$ ), then the data needs to be structured in two dimensions: hour, day. Lastly, data reduction and graphical data analysis must be performed, which is explained in Sections 2.2 and 2.4. This framework allows a more intuitive querying interaction of the sounds database to be built by combining visual selections with pattern enhancement by means of image processing techniques.

Structuring the data in the 2D time frame requires a precise clock without any kind of drift. Although undesirable, time drift sometimes appears. Time drift may be aggravated by file system latency increasing as the number of recorded files increases. Different techniques can be used to correct this drift, but the most simple one is encoding the real date/time into the file header when the 


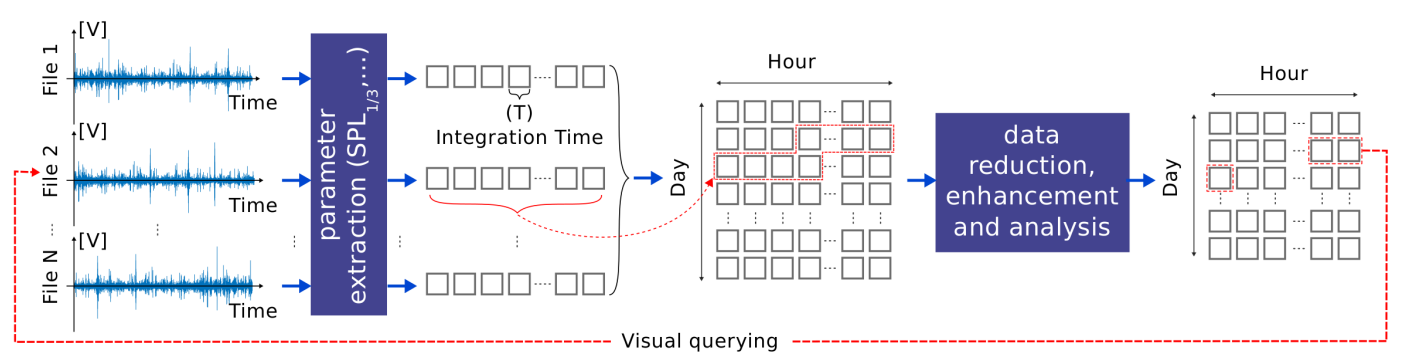

Figure 1: Obtaining the 2D heat map representation from the acoustic signal involves the following steps (among others): extracting the parameter to be represented (e.g., $S P L_{1 / 3}$ ), structuring the data in 2D time frames (Hour, Day), and data reduction for proper representation.

processor is ready to start writing new samples into the file. When applied to SPL indicators, heat map visualizations can represent very long temporal recordings (up to several months) in a very compact way. In addition, they provide a straightforward identification of periodic events or seasonal activities. The main limitation of these representations is the difficulty to visualize more than one variable. Even though comparison between two or more variables is difficult to represent, in the case of a binary variable such as the presence of a given species, this can be done using symbols or semitransparent colors.

A real example of ambient noise indicators such as heat map representations can be found in Figure 2 . The left panel in Figure 2 shows the $S P L_{1 / 3}$ at $2 \mathrm{kHz}$ representation of an acoustic campaign in shallow waters (aprox. 80 meters of depth) done in July 2013 in the Cabrera Archipelago Maritime-Terrestrial National Park (Spain). Among other things, the figure shows coherence in the noise level depending on the time of the day. This produces a characteristic vertical banding effect in the heat map. Sunrise/sunset times were calculated for longitude, latitude, and all deployment dates using a generic astronomy calculator. These sunrise/sunset times have been plotted in Figure 2 (left panel) with a continuous blue line. This reveals a slight increase in the noise level during the night. This is probably due to noise produced by organisms as a result of the diurnal (or diel) vertical migration induced by light. This is a common form of migration where organisms ascend to the surface around dusk, remaining at the surface for the night, then migrating to depth again around dawn. The right panel in Figure 2 shows the $S P L_{1 / 3}$ at $125 \mathrm{~Hz}$ representation of an acoustic campaign done in August 2016 in Cabo de San Antonio Natural Marine Reserve (Denia, Spain). The recorder was placed $4 \mathrm{Km}$ away from the harbour. Among other events, Figure 2 clearly shows the noise increase due to the fishing ves- sels departing at 5:00 AM every week day and returning to the fish market starting at 17:30 PM.

Even though some data reduction is done through the integration time $T$ (see Eq. (1)), further data reduction is needed. The typical integration time used is of the order of [1-20] seconds, which leads to horizontal resolutions greater than those of $4 \mathrm{~K}$ displays $(1440 \mathrm{~min} /$ day $\cdot 3$ pixels $/ \mathrm{min}=4320$ pixels $/$ day that need to be reduced so that heat maps can be visualized in conventional displays. In the two heat maps presented in Figure 2, data was reduced so that, independently of the integration time $T$, each pixel gave an indication of the noise level in a 15-minute period. Our approach for doing such a data reduction should follow an overarching principle: reduced data should be physically meaningful and representative of a large region while at the same time preserving seasonal structures.

\subsection{Data reduction Methods}

Not all of the different data reduction techniques available are suitable when they are used for the assessment of the GES due to acoustic energy emissions. For instance, although straightforward, extracting every $n^{\text {th }}$ point, may lead to the exclusion of some important features in the data such as sharp spikes coming from brief high pressure events. A more feasible alternative is binning aggregation (counting the number of events in a temporal interval). This technique is of direct application when used with detections of animal species that happen during a given time interval. In the case of SPL noise indicators, aggregate data reduction can be done through the histogram and the median or percentile values. The data reduction method used should provide data that is:

- robust to minor changes or differences in implementation. 

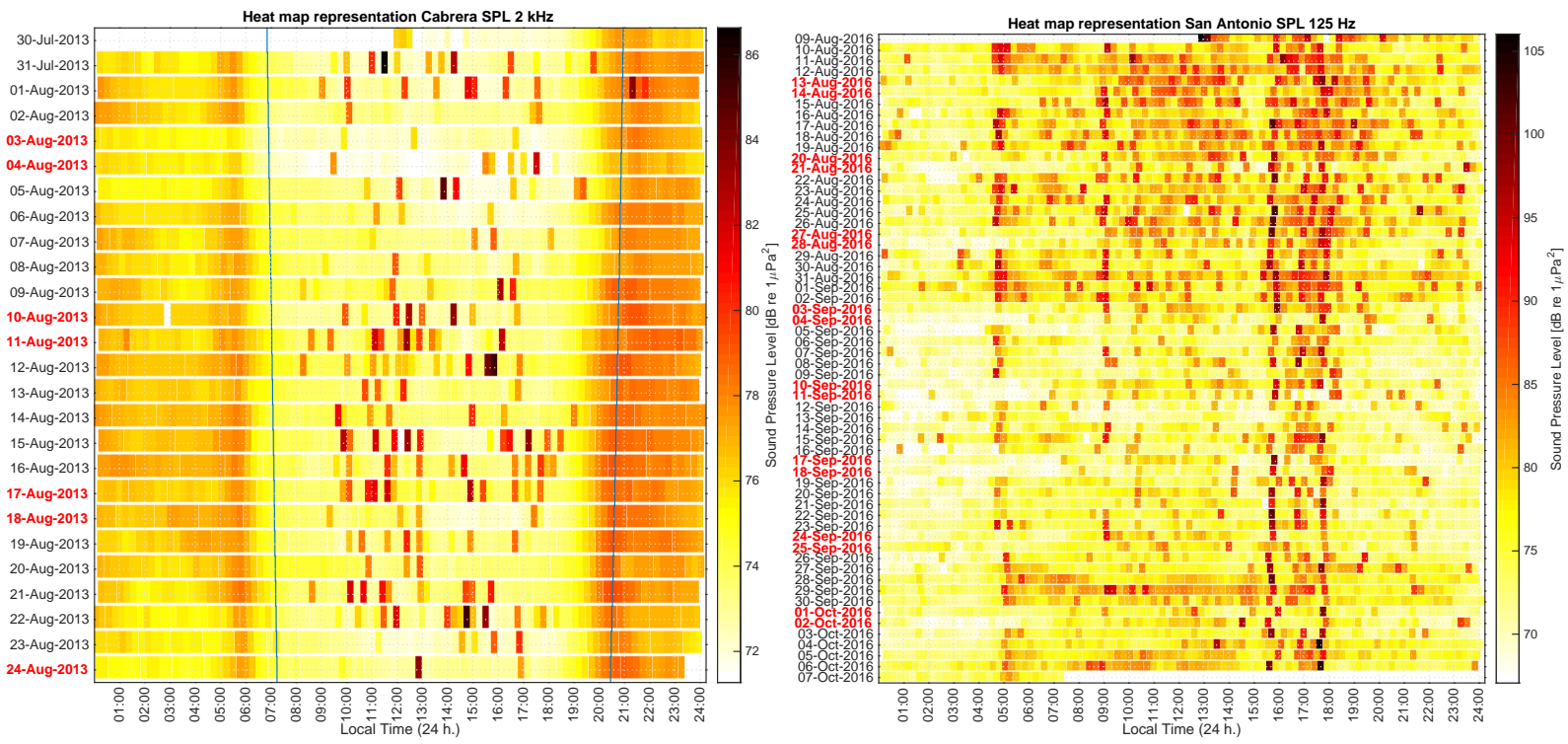

Figure 2: Two examples of a heat map representation of $1 / 3$ octave noise indicators (each pixel gives an indication of the noise level in a 15-minute period). The left panel shows the $S P L_{1 / 3}$ at $2 \mathrm{kHz}$ representation of an acoustic campaign done in July 2013 in the Cabrera Archipelago MaritimeTerrestrial National Park (Spain). The right panel shows the $S P L_{1 / 3}$ at $125 \mathrm{~Hz}$ representation of an acoustic campaign done in August 2016 in Cabo de San Antonio Natural Marine Reserve (Denia, Spain).

- physically meaningful and representative of a large enough region to justify its use as an indicator of GES [12].

- practical (simple to implement).

Figure 3, panel (a) shows a zoomed section of a complete heat map (20-second integration time) alongside a reduced data version using a temporal interval of 15 minutes using: the arithmetic mean (panel (b)), the median (panel (c)), and the statistical noise level $L_{10}$ or percentile $90 \%$ (panel (d)).

Data reduction also includes filtering techniques and modelling techniques as well as hybrid methods or a combination of some of them. For example, the peakpreserving filtering algorithms [13] perform a combination of modeling techniques using straight lines and filtering. Although the computational complexity of some of these techniques may make them inadequate for noise SPL indicators, it is possible to design simple peak-preserving algorithms for short time intervals of data. A peak-preserving data reduction technique for ambient noise indicators should assure that peaks higher than a given threshold are preserved, but at the same time ensure that the average sound pressure level is not increased as happens with the $L_{10}$ data reduction technique described above. This is done in the proposed Algorithm 1. The algorithm starts with fitting a line in a data reduction interval (in our case 15 minutes). Then, it determines whether the data fits the line within a certain tolerance level. If so, the algorithm returns the mean of the data. Otherwise, the data value returned is the data point that is furthest away from the line. The results of the Peak-Preserving Data Reduction Algorithm (PPDRA) are shown in Figure 3, panel (e) where the algorithm is applied to the underwater ambient noise indicator $S P L_{1 / 3}$ in the band of $63 \mathrm{~Hz}$.

$\overline{\text { Algorithm } 1 \text { Proposed simplified peak-preserving data }}$ reduction algorithm

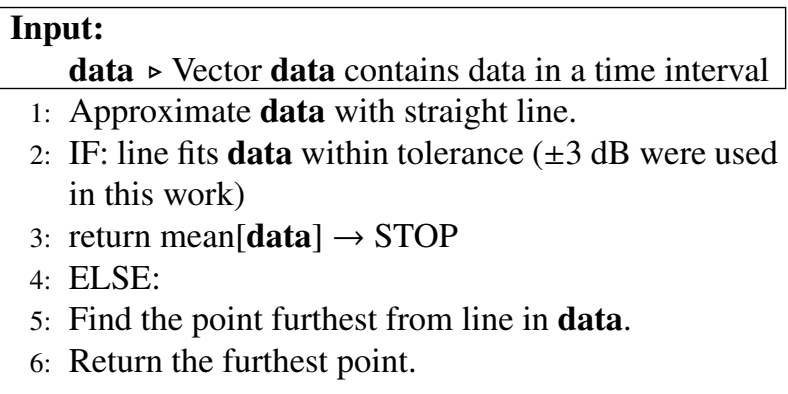

From the Figure 3, it can be observed that arithmetic mean $(3-b)$ and median $(3-c)$ produce similar data reduced representations. The arithmetic mean is sensitive to outliers (extreme high values) that might be caused by 


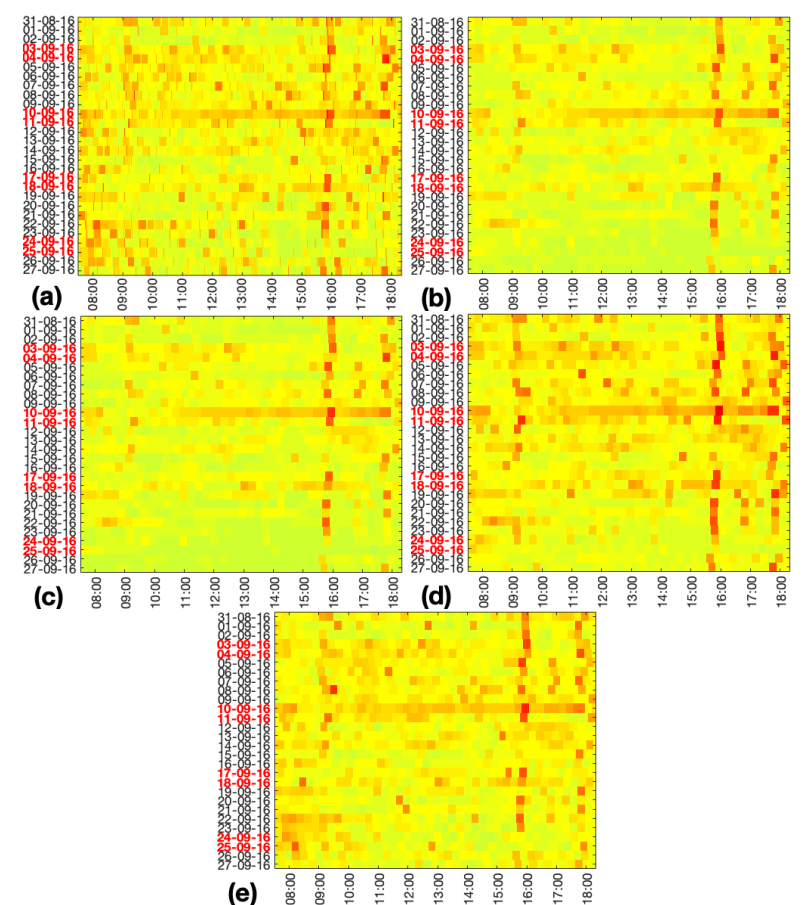

Figure 3: Zoomed region of the $S P L_{1 / 3}$ indicator in the band of 63 $\mathrm{Hz}$. From top left to bottom right: (a) Original representation with 20 -second integration time; (b) data reduced for a 15-minute time interval using the aritmetic mean; (c) data reduced for a 15-minute time interval using the median, (d) data reduced for a 15-minute time interval using $L_{10}$ (percentile $90 \%$ ); and (e) data reduced for a 15-minute time interval using the proposed non-uniform peak-preserving algorithm described.

acquisition or processing artefacts [14]. However, the median tends to exclude some isolated high amplitude excursions that might contribute to total noise. The $L_{10}$ reduction $(3-d)$ preserves some of these excursions but at the same time overestimates the total noise. Finally, the proposed PPDRA technique (3 - e) preserves high amplitude excursion (controlled by the corresponding tolerance parameter in Algorithm 1) while at the same time producing a less biased representation of the total noise than that obtained with the $L_{10}$. Although not presented here, it is important to note that these differences are not so obvious for high frequencies indicators ( $S P L_{1 / 3}$ at $125 \mathrm{~Hz}$ and $2 \mathrm{kHz}$ ).

\subsection{Graphical representation enhancement: Colormap encoding for the detection of seasonal and periodic events}

Periodic or seasonal events might appear as small variations in the SPL values that can be difficult to detect within the whole range of variation of the acoustic index. In some situations, color encoding can enhance and ease the visibility of seasonal patterns in heat map representation (equivalent to some sort of heat map equalization). Traditional image equalization techniques sometimes give good results in increasing the contrast in vertical structures, but care must be taken since some of these techniques introduce a non-linearity in the histogram that might make the physical interpretation of the SPL difficult. In our work, we propose mapping an acoustic SPL to a pseudo-color value $y \in[0,1]$ using the formula:

$$
y=\frac{\widehat{S P L_{1 / 3}}-S P L_{1 / 3}^{\min }}{S P L_{1 / 3}^{\max }-S P L_{1 / 3}^{\min }}
$$

where $\widehat{S P L_{1 / 3}}$ is the value of $S P L_{1 / 3}$, bounded from above and below by the range parameters $S P L_{1 / 3}^{\max }$ and $S P L_{1 / 3}^{\min }$. These parameters can be interactively selected by the user or automatically adjusted to optimize the contrast of the heat map.

\subsection{Graphical representation analysis: Automatic de- tection of vertical structures}

One of the main applications of heat maps in ambient noise monitorization is the detection of seasonal events or events happening with a given periodicity (daily, weekly, etc.). If the heat map is conveniently structured, these periodic events are shown as vertical lines in the heat map. Different line detection algorithms may be used. The Hough transform is a feasible alternative. However, care must be taken since this transformation was not devised for graphs. The data reduction stage produces aggregated bins that might have rectangular shape (having different pixel sizes in the horizontalvertical dimensions). This situation, might be interpreted by the Hough transform as horizontal/vertical lines. Transforming rectangular bins into square ones (with the same number of pixels in both dimensions) and applying a threshold to obtain a binary black and white heat map representation is necessary for proper identification of vertical structures. Let $I(x, y)$ be a heat map representation. The heat map can be binarized by applying a convenient threshold $\tau$ according to Eq. (3).

$$
B_{\tau}(x, y)= \begin{cases}1, & \text { if } I(x, y) \geq \tau \\ 0, & \text { if } I(x, y)<\tau\end{cases}
$$

If we define $\mathcal{H}[$.$] as the operator that transforms a$ binary image into the $\theta-\rho$ space, we can obtain the Hough transform $\left(H_{\tau}(\theta, \rho)\right)$ according to Eq. (4).

$$
H_{\tau}(\theta, \rho)=\mathcal{H}\left[B_{\tau}(x, y)\right]
$$




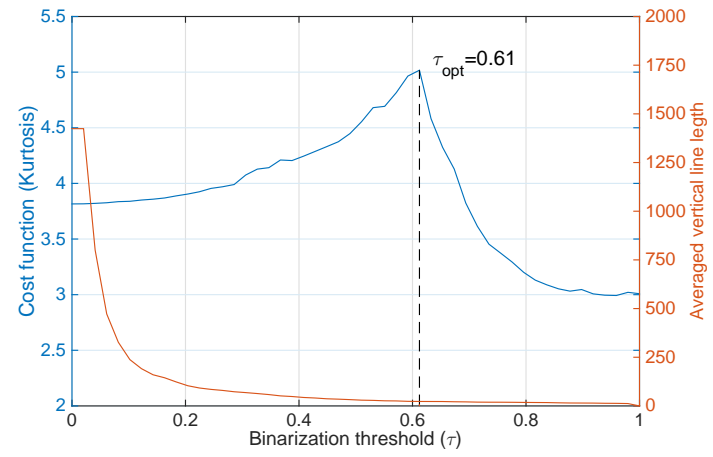

Figure 4: Evaluation of the cost function for different threshold levels (averaged over 100 Monte Carlo runs). The graph also shows the mean vertical line length detected using the Hough transform.

Looking for vertical lines implies looking for local maximum in $H_{\tau}(\theta, \rho)$ when $\theta=0$. It is then only necessary to compute $H_{\tau}(0, \rho)$, thus reducing the computational complexity of the Hough transform algorithm. Additionally, we can use some "shape factor" obtained from $H_{\tau}(0, \rho)$ as a cost function to be maximized in order to automatically adjust the binarization threshold $(\tau)$ of $I(x, y)$. An approach that has proven to work in this particular situation is described in Eq. (5)

$$
\tau_{o p t}=\underset{\tau}{\operatorname{argmax}}\left\{\operatorname{Kurt}\left[H_{\tau}(0, \rho)+\{\tilde{n}(\rho)\}\right]\right\}
$$

, where Kurt[.] is the kurtosis and $\{\tilde{n}(\rho)\}$ is a low-level Gaussian noise $\left(10 \%\right.$ of the maximum $\left.H_{\tau}(0, \rho)\right)$ that is added to avoid indetermination when trying to compute the kurtosis of constant values such as those obtained for very low or high thresholds. The kurtosis was chosen to generate the cost function to be maximized since, under some circumstances, it may be representative of the peakedness of the distribution. The presence of local maxima in the $\theta-\rho$ space at $\theta=0$ produces a distribution with heavy tails, thus increasing the value of the kurtosis. Figure 4 shows the evolution of the cost function and the average vertical line length (in pixels) for a Monte Carlo simulation of heat map representation with three daily repeating seasonal events.

The different stages of the process are illustrated in Figure 5. Starting with a gray level heat map graph, successive binarizations are done for a range of thresholds Eq. (3). The maximization of Eq. (5) allows us to obtain the optimum threshold. The right top panel in Figure 5), shows the binarized heat map for $\tau_{o p t}$ and the bottom panel shows its Hough transform. The local maxima of the Hough transform at $\theta=0$ (highlighted

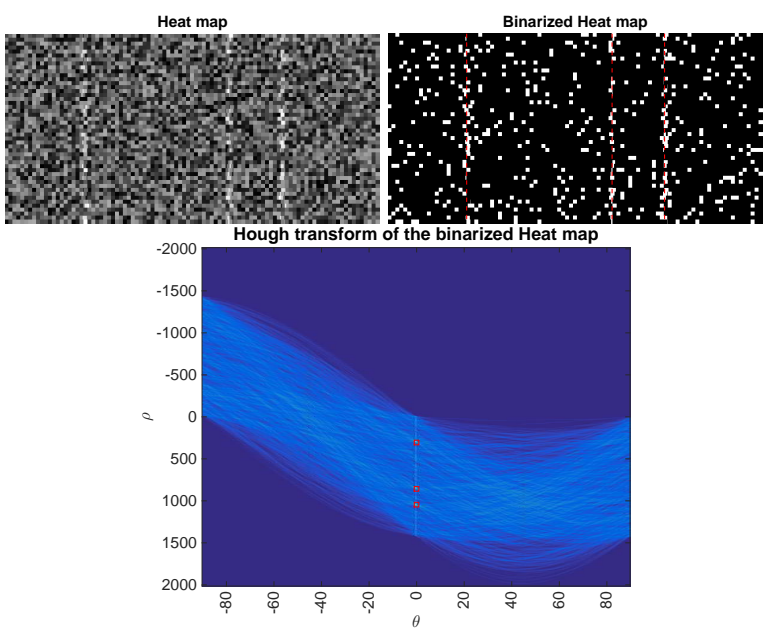

Figure 5: From top left to bottom right: original heat map, binary heat map, Hough transform of the heat map. The three red dots in $\theta=0$ correspond to the three daily repeating events seen in the original heat map.

with red squares in the bottom panel) reveals the existence of daily repeating events.

Despite the huge potential of the proposed heat map representations, it is sometimes desirable to be able to represent two or more variables in order to establish correlations among them. For instance, in ambient noise monitoring it is interesting to be able to simultaneously represent $1 / 3$ octave band SPL indicators alongside cetacean species detection.

\subsection{Two-layer structure for the detection of cetacean presence}

There are different alternatives for the representation of more than one variable in the described heat map representation of an acoustic monitoring campaign. One possible technique consists in encoding one of the variables in the luminance and using the chrominance to represent the other variable (YUV colorspace). This technique should be restricted to situations were the variable to be encoded in the chrominance is a binary variable (e.g., the presence / absence of cetacean sounds). Otherwise, the final representation may be difficult to understand. Eq. (6) and Eq. (7) show how we can create a two-layer representation, for a given binary variable $a \in\{0,1\}$ and a sound pressure level $S P L_{1 / 3}$, by selecting a color $\left[u_{1}, v_{1}\right]$ in the UV color plane and a transformation $\Gamma$ that maps pressure levels in the luminance (Y).

$$
\left[\begin{array}{l}
U \\
V
\end{array}\right]=\left[\begin{array}{l}
u_{1} \\
v_{1}
\end{array}\right] \cdot a
$$




$$
Y=\Gamma\left(S P L_{1 / 3}\right)
$$

The left panel of Figure 6 illustrates this idea of encoding the ambient noise indicator in the luminance and the detection of cetaceans in the chrominance $\left[u_{1}, v_{1}\right]=$ $[43,21]$ (green bins).

The other technique that might also be used is much more simple. It consists in using different symbols overlaid in the heat map representation. As an example, the right panel of Figure 6 shows how a rectangular box can be overlaid to indicate the presence of cetacean sounds.

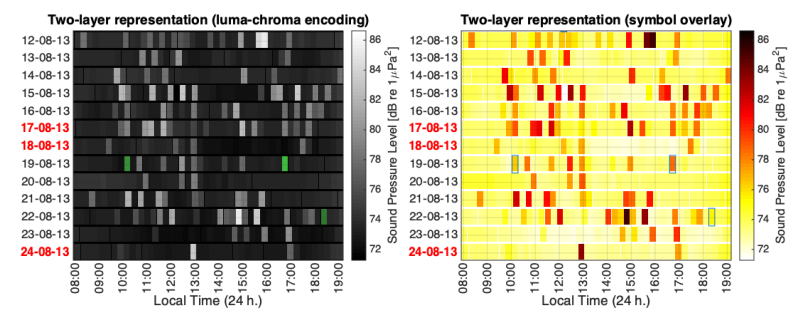

Figure 6: Representation of two variables in a heat map. The left panel: luminance heat map with chrominance encoding. The right panel: heat map with overlay of rectangular symbols.

\section{Enhanced representation of seasonal events: Po- lar Area or Coxcomb Diagrams}

Polar area diagrams are an interesting tool for representing the cyclical nature in time series of data - a season in a year, a time of the day, and so on. A polar diagram looks like a traditional pie chart, but the sectors do not differ from each other by the size of their angles but rather by how far they extend out from the centre of the circle. As a result, they are an excellent tool for the characterization of daily events or seasonal occurrences. We can also use these diagrams to represent more than one variable, thus avoiding the limitation of one of the variables being binary. To the best of our knowledge, polar area diagrams have never been used to represent underwater ambient noise indicators or as a tool to evaluate the presence of anthropogenic noise.

The creation of ambient noise polar area diagrams can be easily done from the heat maps described in the previous section. Hourly marginal distributions can be computed from the heat map matrix (see Figure 1) for all days or just for a selection of them. In this way, any noise indicator (e.g., $S P L_{1 / 3}$ at $63 \mathrm{~Hz}$ ) can be plotted in polar coordinates as a function of the hour of the day. As an example, we represent the data introduced in Section 2 from Cabo de San Antonio Natural Marine
Reserve. We have divided the heat map matrix data into two different sub-matrices: the first matrix corresponds to weekdays, whereas the second matrix corresponds to weekends. The standard ambient noise indicators in each of the $1 / 3$ octave frequency bands $(63,125$, and $2000 \mathrm{~Hz}$ ) were computed daily and averaged for each one of the two sub-matrices. To avoid misrepresentation, it is important to highlight that in the successive polar representations we used a sector radius (and not a sector area) that was proportional to the variable being represented.

Figure 7 represents the polar area diagram of the different noise indicators: the top panel represents the hourly SPL noise distribution during the weekend and the bottom panel represents the hourly SPL noise distribution during weekdays. In both panels, we have included the sunrise and sunset variations within the deployment period as a light gray sector. The representation gives a clear indication of the cyclic nature of the represented variable while at the same time comparing different ambient noise indicators. As shown in Figure 7 , the daily repeating events that were produced during weekdays by shipping activity were clearly identified. In addition, the polar area diagrams also show peaks at 9:00 AM with high intensity during weekdays (85 dB re $1 \mu \mathrm{Pa}$ at $125 \mathrm{~Hz}$ ) and low intensity during weekends ( $80 \mathrm{~dB}$ re $1 \mu P a$ at $125 \mathrm{~Hz}$ ). This is due to the ferry that travels from Denia to Ibiza. Two more peaks at 15:46 and 17:46 happening daily can also be identified in the diagrams. It is also interesting to highlight how during the night and in the absence of anthropogenic noise, ambient noise levels go back to a situation where noise levels decrease with increasing frequency $\left(S P L_{1 / 3} 63\right.$ $\left.\mathrm{Hz}>S P L_{1 / 3} 125 \mathrm{~Hz}>S P L_{1 / 3} 2 \mathrm{kHz}\right)$. The relationship among the three ambient noise levels may serve as a human activity indicator.

\section{Conclusions}

We have proposed a graphical visualization framework that is advantageous when working with large databases of sound files. This framework has been applied to passive acoustic monitoring of underwater sounds, specifically to ambient noise and cetacean presence indicators. As a result of this work, two different visualization techniques are proposed. The first technique, which is a variant of heat maps, is very suitable for representing very long temporal series. It provides a global acoustic panorama while at the same time revealing the presence of short duration events as well as different kinds of seasonal activities. We also studied the 


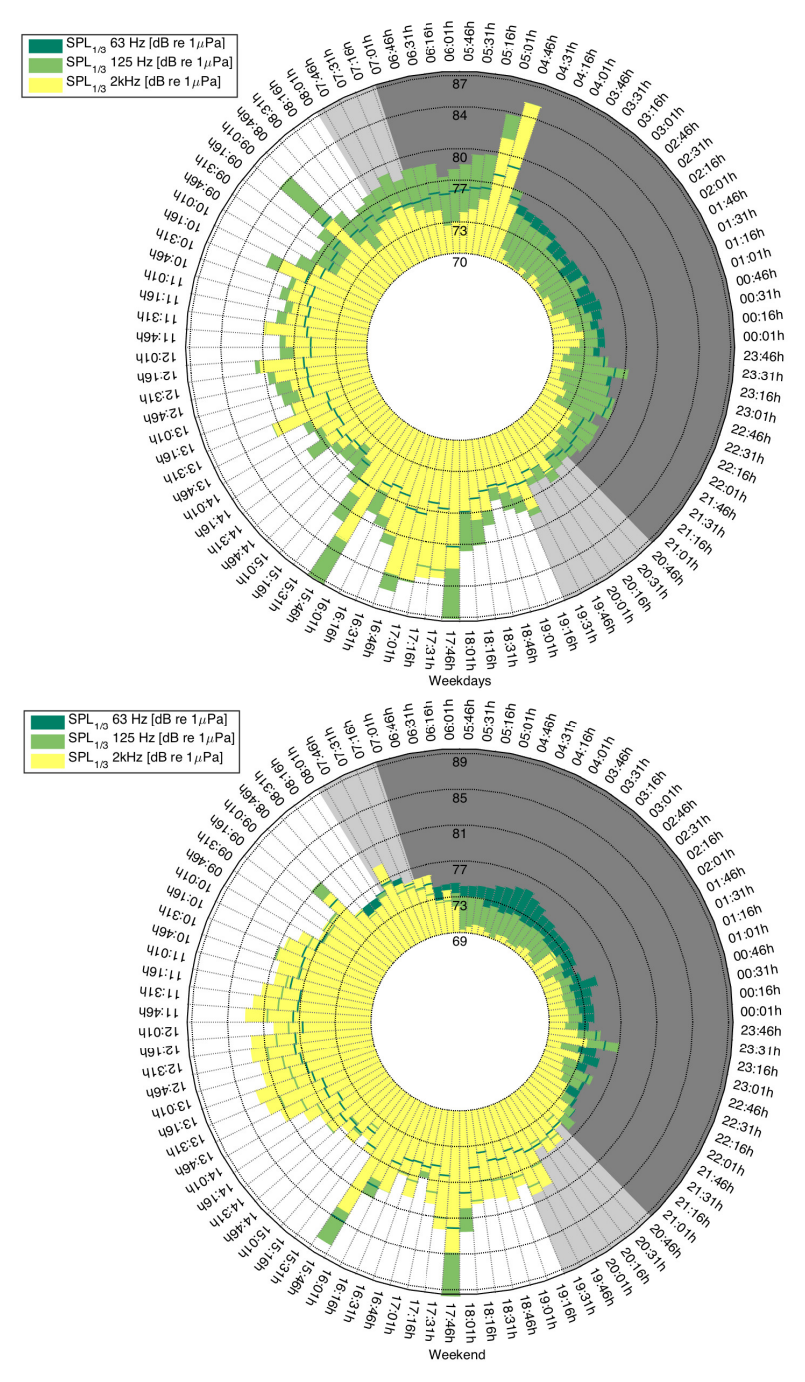

Figure 7: Example of a coxcomb chart for the representation of $1 / 3$ octave noise indicators and their daily variation in the Cabo de San Antonio Natural Marine Reserve.

influence of some variables such as data reduction techniques and automatic detection of vertical structures. Based on this study, we have presented an algorithm to reduce the volume of data in order to adapt it to the resolution of conventional displays and an algorithm to detect seasonal structures. The proposed representation can also be used for visual querying of a large database of recordings.

A second visualization technique, which is created by means of polar area diagrams, has been devised using marginal distributions of the heat map representations. Although it is not very suitable for big data visualization, it has great potential when performing a detailed analysis of several variables under different circumstances. An example is given to illustrate how noise from shipping lanes changes during the weekdays/weekends and how precise detection of ship departures times can be determined.

Both proposed techniques can be used to evaluate circadian rhythms as we have illustrated with the heat map representations. Although the proposed framework has been applied to the evaluation of ambient noise and its possible influence on the behavior of some animal species, the work presented here may be of interest in many other areas such as the design of surveillance systems or the visualization and analysis of natural phenomena.

\section{Acknowledgements}

This work was supported partially by the Fundación Biodiversidad (various Spanish government ministries involved in nature conservation) and partially by the European Commission - DG ENV (grant agreement 11.0661/2016/748066/SUB/ENV.C2). We would like to thank all of the researchers from FGUA (UAH) and the Oceanogràfic (Valencia) who participated in the deployment and recovery of the acoustic recording devices. We also thank the authorities in charge of the Cabrera Archipelago Maritime-Terrestrial National Park as well as the Cabo de San Antonio Natural Marine Reserve for providing the corresponding permits to carry out the scientific work.

\section{References}

[1] J. Finneran, Noise-induced hearing loss in marine mammals: A review of temporary threshold shift studies from 1996 to 2015 , The Journal of the Acoustical Society of America 138 (3) (2015) 1702-1726. doi:10.1121/1.4927418.

[2] H. Kunc, K. McLaughlin, R. Schmidt, Aquatic noise pollution: implications for individuals, populations, and ecosystems, Proceedings of the Royal Society B: Biological Sciences 283 (1836) (2016) 1-8. doi:10.1098/rspb.2016.0839.

[3] V. der Graaf AJ, M. Ainslie, M. Andre, K. Brensing, J. Dalen, R. Dekeling, S. Robinson, M. Tasker, F. Thomsen, S. Werner, European Marine Strategy Framework Directive - Good Environmental Status (MSFD-GES), Report of the technical subgroup on underwater noise and other forms of energy (2012) $1-75$.

[4] A. Borja, M. Elliott, P. V. R. Snelgrove, M. C. Austen, T. Berg, S. Cochrane, J. Carstensen, R. Danovaro, S. Greenstreet, A.S. Heiskanen, C. P. Lynam, M. Mea, A. Newton, J. Patrício, L. Uusitalo, M. C. Uyarra, C. Wilson, Bridging the Gap between Policy and Science in Assessing the Health Status of Marine Ecosystems, Frontiers in Marine Science 3 (2016) 1-23. doi:10.3389/fmars.2016.00175.

[5] R. Dekeling, M. Tasker, A. V. der Graaf, M. Ainslie, M. Andersson, M. Andre, J. Borsani, K. Brensing, M. Castellote, 
D. Cronin, J. Dalen, T. Folegot, R. Leaper, J. Pajala, P. Redman, S. Robinson, P. Sigray, G. Sutton, F. Thomsen, S. Werner, D. Wittekind, J. Young, Monitoring guidance for underwater noise in European seas, Part II: Monitoring Guidance Specifications, JRC Scientific and Policy report EUR 26555 EN, Publications Office of the European Union, Luxembourg (2014) 1-49.

[6] J. Johansson, P. Ljung, M. Jern, M. Cooper, Revealing structure within clustered parallel coordinates displays, in: IEEE Symposium on Information Visualization, 2005. INFOVIS 2005., 2005, pp. 125-132. doi:10.1109/INFVIS.2005.1532138.

[7] M. F. Baumgartner, S. E. Mussoline, A generalized baleen whale call detection and classification system, The Journal of the Acoustical Society of America 129 (5) (2011) 2889-2902. doi:10.1121/1.3562166.

[8] T. A. Helble, G. R. Ierley, G. L. D'Spain, M. A. Roch, J. A. Hildebrand, A generalized power-law detection algorithm for humpback whale vocalizations, The Journal of the Acoustical Society of America 131 (4) (2012) 2682-2699. doi:10.1121/1.3685790.

[9] J. N. Oswald, S. Rankin, J. Barlow, M. O. Lammers, A tool for real-time acoustic species identification of delphinid whistles, The Journal of the Acoustical Society of America 122 (1) (2007) 587-595. doi:10.1121/1.2743157.

[10] D. M. Gillespie, O. Chappell, An automatic system for detecting and classifying the vocalisations of harbour porpoises, Bioacoustics 13 (1) (2002) 37-61. doi:10.1080/09524622.2002.9753485.

[11] Z. Liu, B. Jiang, J. Heer, imMens: Real-time visual querying of big data, Eurographics Conference on Visualization (EuroVis) 32 (3) (2013) 1-10.

[12] A. N. Popper, A. D. Hawkins (Eds.), The effects of noise on aquatic life II, no. 875 in Advances in experimental medicine and biology, Springer, New York, 2012, Ch. 24.

[13] M. C. Hao, H. Janetzko, S. Mittelstädt, W. Hill, U. Dayal, D. A. Keim, M. Marwah, R. K. Sharma, A Visual Analytics Approach for Peak-Preserving Prediction of Large Seasonal Time Series, Computer Graphics Forum 30 (3) (2011) 691-700. doi:10.1111/j.1467-8659.2011.01918.x.

[14] R. Dekeling, M. Tasker, A. V. der Graaf, M. Ainslie, M. Andersson, M. Andre, J. Borsani, K. Brensing, M. Castellote, D. Cronin, J. Dalen, T. Folegot, R. Leaper, J. Pajala, P. Redman, S. Robinson, P. Sigray, G. Sutton, F. Thomsen, S. Werner, D. Wittekind, J. Young, Monitoring guidance for underwater noise in European seas, Part III: Background Information and Annexes, JRC Scientific and Policy report EUR 26555 EN, Publications Office of the European Union, Luxembourg (2014) 172. 